\title{
Experimental study of the potential use of diffusing wave spectroscopy to investigate the structural characteristics of blood under multiple scattering
}

\author{
Alexander N. Korolevich a,b, *, Igor V. Meglinsky ${ }^{\mathrm{c}, \mathrm{d}}$ \\ ${ }^{a}$ Departamento de Fisica, Universidade do Minho, Campus de Gualtar, 4709 Braga, Portugal \\ ${ }^{\mathrm{b}}$ A.I. Stepanov Institute of Physics, National Academy of Science of Belarus, Skarina Ave., 68, 220072, Minsk, Belarus \\ ${ }^{\mathrm{c}}$ School of Physics, University of Exeter, Stocker Road, Exeter EX4 4QL, UK \\ ${ }^{d}$ Department of Physics, Saratov State University, Astrakhanskaia Str., 83, Saratov 410026, Russian Federation
}

\begin{abstract}
The extension of the photon correlation spectroscopy (PCS) in multiple scattering regime, so-called diffusing wave spectroscopy (DWS) was employed to the study of blood samples. Multiple scattered light from a helium-neon (He-Ne) laser beam incident on the blood samples was detected by a photomultiplier, and both the temporal autocorrelation intensity functions $g_{2}(\tau)$ and power spectra $S(\omega)$ were measured by a spectrum analyzer. The potentials of using DWS for the qualitative and quantitative determination of the structural characteristics of the blood elements were studied experimentally. The experimental studies made, permits the use of DWS for blood cells monitoring in a multiple scattering regime. This paper describes our initial attempts at applying DWS to the study of the discrete blood samples of both healthy donors and patients with the cardiac ischemia. The subsequent experiments provide a verification of DWS of blood cells shape monitoring under multiple scattering.
\end{abstract}

Keywords: Diffusing wave spectroscopy; Blood; Multiple scattering; Temporal field autocorrelation function

\section{Introduction}

The unique characteristics of light scattering methods are of great interest to researchers working in different areas of biology and medicine [1-3]. Nevertheless, the problem of implementing optical diagnostic methods in clinical practice in order to solve a wide range of actual diagnostic tasks remains. Part of the reason for this can be explained by the fact that for most biological tissues, probing radiation is usually in the visible and near-infrared ranges of the spectrum $(\lambda=400-1400 \mathrm{~nm})$. This region is characterized by low absorption, and highly and often anisotropy scattering [4]. As a result, the light waves emerging from the processes of diffraction and scattering

\footnotetext{
* Corresponding author. Departamento de Fisica, Universidade do Minho, Campus de Gualtar, 4709 Braga, Portugal. Fax: +351-253-678981.

E-mail address: akaralevich@fisica.uminho.pt (A.N. Korolevich).
}

by the structural elements of the medium being investigated have different phase constituents that cause interference phenomena. The mathematical expression for the propagation of radiation which describes these conditions is complex. In practice, it is not possible to distinguish regular waves corresponding to the internal structure of the medium or individual characteristics of scatters.

It has been demonstrated that the analysis of the dynamics of scatters and internal media structure by the use of photon correlation spectroscopy (PCS) is a very promising technique $[3,5,6]$. Moreover, the recent extension of PCS to a multiple scattering regime, known as diffusing wave spectroscopy (DWS) [7,8], to monitor the microscopic displacements of the scattering particles (up to hundredths of a wavelength $\lambda$ of the incident radiation) [9], and its successful application for in vivo blood flow diagnostics $[10,11]$ have initiated the current experimental study of the structural and biochemical characteristics of blood. These characteristics are very important indicators for both the general state of the human body and the functioning of its 
individual organs. There are up to 40 parameters (proteins, microelements, globulins, etc.), whereas typically for the particular diseases, only four or five are of interest. We investigated the blood of patients with cardiac ischemia and therefore, checked the lipoprotein concentration, degree of oxygenation, average size of the aggregates and the shape of the red blood cells.

Thus, taking into account the multi-parametric characteristic of the problem, we have investigated the multiple scattering of the laser radiation depending on the change of the biochemical and rheological characteristics of human blood samples.

\section{Principles of the diffusing wave spectroscopy}

Despite the similarity of DWS and PCS experimental setups $[5,6]$, the DWS is based on quite different interpretation of the results. Thus, in a framework of DWS, the radiation propagation is described as a diffusion of photons through the inhomogeneous turbid highly scattering medium $[7,8]$.

Due to multiple scattering, each photon undergoes a large number of scattering events, $N$, and a resultant phase shift $\Delta \phi(\tau)[7]$ :

$\Delta \phi(\tau)=\sum_{i=1}^{N} \boldsymbol{q}_{i}\left[r_{i}(t+\tau)-r_{i}(t)\right]=\sum_{i=1}^{N} \boldsymbol{q}_{i} \Delta r_{i}(\tau)$,

where, $\boldsymbol{q}_{i}$ is the scattering wave vector for the $i$-th scattering event, $r_{i}(t)$ and $r_{i}(t+\tau)$ are the coordinates of the scatterers in time $t$ and $t+\tau$, respectively and $\Delta r_{i}(t)$ is the position change of the $i$-th scattering particle during time $\tau$. It should be noted that in highly scattering random medium, the trajectory $s$ of each photon is an independent random walk from the source $r_{0}$ to the detector points $r_{N+1}$.

Thus, at an instance of time $t$ due to sequential scattering by a series of scatterers $r_{1}(t), r_{2}(t), \ldots, r_{N}(t)$ in a far zone performs a scattering field $E(t)[12,13]$ :

$E(t)=\sum_{i=1}^{N} \gamma_{i} A_{i}(t) \exp \left(i \omega_{0} t+i \phi_{i}(t)\right)$

where $|\gamma|^{2}=\sin \psi, \psi$ is the angle between direction of the polarization vector and direction of scattering, $A_{i}(t)$

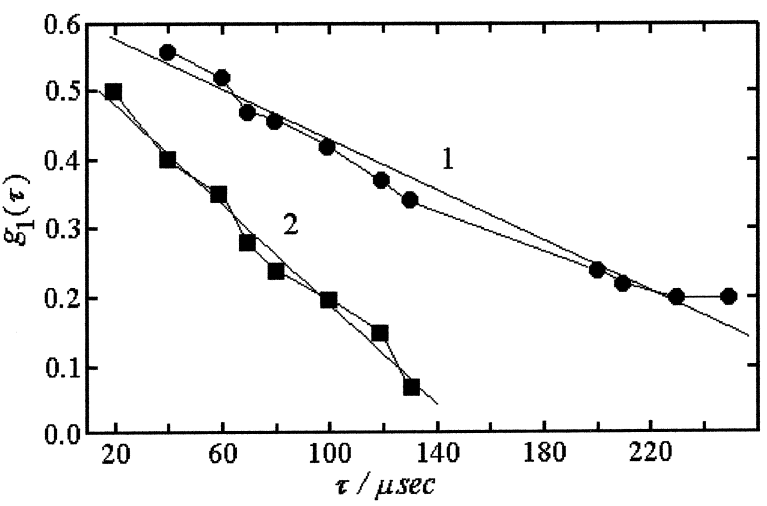

Fig. 2. Normalized temporal field autocorrelation functions $g_{1}(\tau)$, measured for two different particle shaped samples: 1-disc-shaped (average diameter of particles $7.2 \mu \mathrm{m}$, and the parameter of aspherity $p=3.3$ ) and 2 - spherical erythrocytes (average diameter of particles $5.6 \mu \mathrm{m}$, and $p=1$ ). The solid lines are the results of linear fitting for the fragments of the temporal field autocorrelation functions.

are the complex amplitudes of scattered waves, $\omega_{0}$ is the frequency of the incident wave.

The field $E(t)$ is interference with the fields $E(t \pm \tau)$ scattered slightly earlier or later on the same series of the scatters. However, in the result of the scattering particles motion in the medium, the phase between fields $E(t)$ and $E(t \pm \tau)$ will be different at different instances of time. The random temporal phase fluctuations predetermine the temporal fluctuations of a summarized field and intensity of scattering radiation, respectively.

Quantitatively, these fluctuations are best examined by the comparative analysis of the temporal field correlation functions of the scattering radiation $[7,8]$ :

$$
\begin{aligned}
G_{1}(\tau) & =\langle E(t) E(t+\tau)\rangle \\
& =I_{0} \sum_{j=0, \infty} P\left(s_{j}\right) \exp \left(-\frac{1}{3} k_{0}^{2}\left\langle\Delta r^{2}(\tau)\right\rangle \frac{s_{j}}{l}\right),
\end{aligned}
$$

where $I_{0}=\left\langle|E(t)|^{2}\right\rangle, l$ is the scattering mean-free path, and $P(s)$ is the normalized distribution of the path lengths $[7,8]$ :

$$
P\left(s_{i}\right)=\left(\frac{v}{4 \pi s_{i} D}\right)^{3 / 2} \exp \left(\frac{v\left|r_{0}-r_{N+1}\right|^{2}}{4 s_{i} D}\right),
$$

where $D=v l / 3$ is the photon diffusion coefficient, and $v$ is the speed of light in the medium.

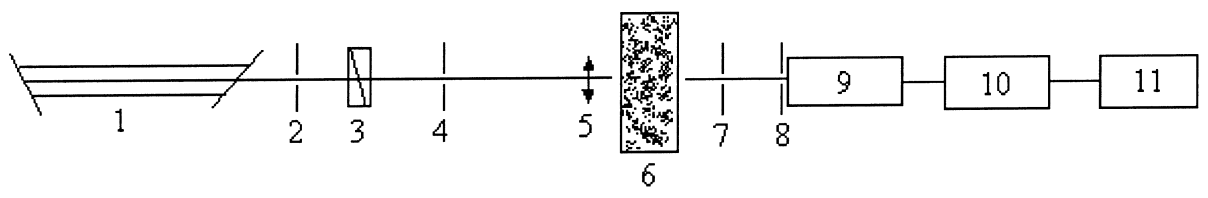

Fig. 1. Schematic diagram of the experimental set-up. 1-He-Ne laser, 2, 4, 7-confining diaphragms, 3-polarizer; 5-focusing lens; 6-cell with a blood sample; 8-the photodetector diaphragm; 9-photomultiplier; 10—spectra analyzer or correlator; 11—two-coordinate recorder. 
Table 1

The $\Delta \omega$ halfwidth of spectrum with respect to the average shape of erythrocytes and surface relief

\begin{tabular}{lllll}
\hline $\begin{array}{l}\text { Shape of } \\
\text { particles }\end{array}$ & Disk & $\begin{array}{l}\text { Relieved } \\
\text { disk }\end{array}$ & Spheroid & Sphere \\
\hline$\Delta \omega / \mathrm{Hz}$ & $333 \pm 23$ & $429 \pm 30$ & $375 \pm 26$ & $273 \pm 19$ \\
\hline
\end{tabular}

On the other hand, the shape of the arbitrary distributed particles during their motion may change in time and the amplitude $A(t)$ of scattered field (2) will change in time as well, respectively. The temporal field correlation function in this case can be considered as:

$G_{1}(\tau) \propto G_{A}(\tau) G_{\phi}(\tau)$,

where $G_{A}(\tau)=\langle A(t) A(t+\tau)\rangle$ is the temporal amplitude correlation function, and $G_{\phi}(\tau)=\langle\phi(t) \phi(t+\tau)\rangle$ is the temporal phase correlation function.

Thus, the normalized temporal field correlation function is responsible for both the dynamics of the scatters, their orientation, and their shape changes. Moreover, for identical dynamics, the temporal field correlation function is characterized by the particle shape changes.

\section{Experimental}

The measurements of the intensity fluctuations of the coherent laser radiation multiple scattering in a sample of the whole human blood were made by the standard PCS experimental scheme $[5,6]$. Experimental setup is shown schematically in Fig. 1. A wavelength of $\lambda=632.8 \mathrm{~nm}$ generated by the $\mathrm{He}-\mathrm{Ne}$ laser was focused on the surface of a spectrophotometer cell $(30 \mathrm{~mm}$ in diameter and $4 \mathrm{~mm}$ in thickness). The diameter of the beam waist in the cell was about $150 \mu \mathrm{m}$, and the power of the incident radiation on the surface of the sample was about $15 \mu \mathrm{W}$.

The subject of our study is the whole blood, which in terms of optics is a heterogeneous liquid, comprising such scattering particles such as erythrocytes (45\%), leukocytes and trombocytes (1\%), and plasma [14]. We assume that the blood characteristics are roughly determined by the characteristics of erythrocytes and plasma alone.

Diffusely scattered light selected by the series of confining diaphragms was collected by a photomultiplier. The output photomultiplier signal was fed into spectrum analyzer or correlator, and the power spectra $S(\omega)$ or temporal intensity autocorrelation functions $g_{2}(\tau)$ were recorded with a two-coordinate recorder.

Both these quantities $S(\omega)$ and $g_{2}(\tau)$ relate to $g_{1}(\tau)$ according to the Wiener-Kintchine theorem [5,7]:

$S(\omega)=\frac{\langle I\rangle^{2}}{2 \pi} \int_{-\infty}^{+\infty}\left[g_{1}(\tau)\right]^{2} \cos \left(i \omega_{0} \tau\right) \mathrm{d} \tau$,

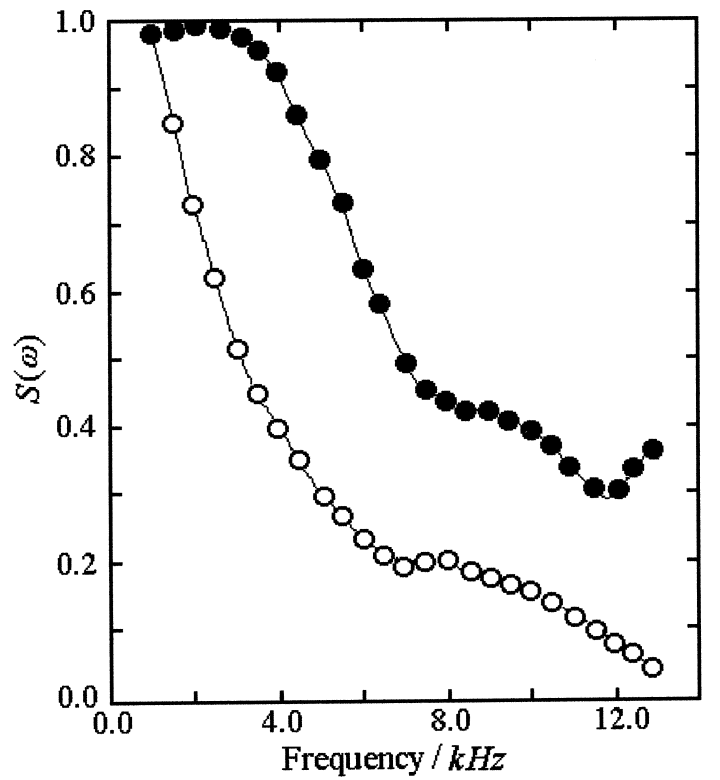

Fig. 3. Spectrum of the intensity fluctuation of the radiation scattered by the blood samples with: $\bigcirc$ - high $(70-150 \mu \mathrm{m})$ and - -low (15-25 $\mu \mathrm{m})$ degree of the erythrocytes aggregation.

and to the Sigert relation temporal intensity autocorrelation function [5]:

$g_{2}(\tau)=1+\beta\left|g_{1}(\tau)\right|^{2}$,

where $\beta$ is the aperture function [7].

\section{Results and discussion}

Fig. 2 presents the typical normalized autocorrelation functions of the scattered radiation field $g_{1}(\tau)$, measured

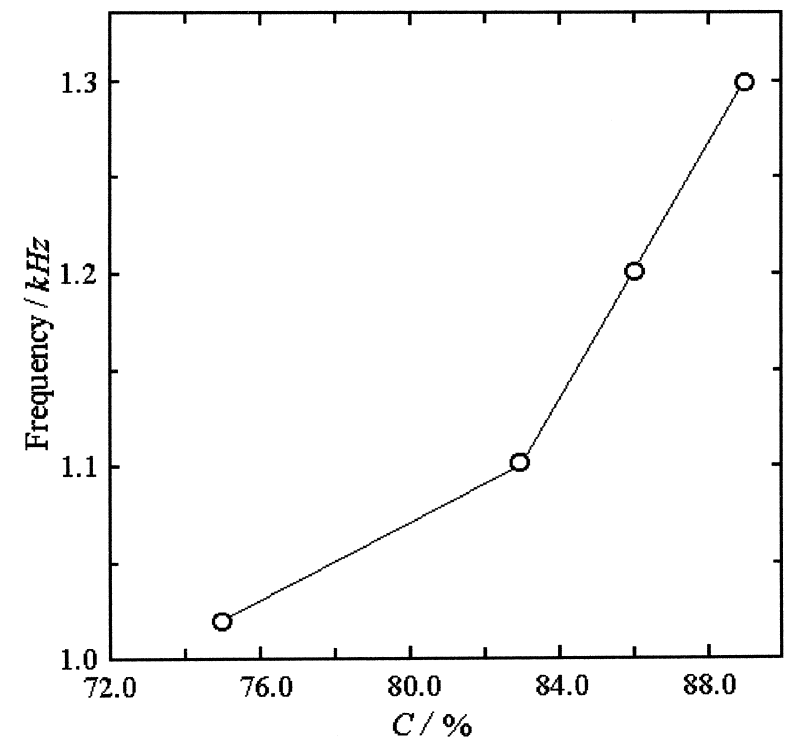

Fig. 4. Halfwidth of a spectrum fluctuation of intensity as a function of the concentration of oxygenium in erythrocytes $(\mathrm{C} \%)$. 
Table 2

Scattered radiation halfwidth of spectrum $\Delta \omega$ vs. the particle shape with the related values of $\alpha$ [18]

\begin{tabular}{llr}
\hline Particle shape & $\Delta \omega / \mathrm{Hz}$ & \multicolumn{1}{c}{$\alpha$} \\
\hline Ferulized erythrocytes & $401 \pm 28$ & 870 \\
Flat disc & $546 \pm 38$ & 2330 \\
Flat disc & $377 \pm 26$ & 28 \\
\hline
\end{tabular}

for disc-shaped and spherical human blood erythrocytes under a constant volume. These results agree well with model experiments using solutions of latex in water [15].

The results of the power spectrum $S(\omega)$ measurements for the blood samples with various average shapes of erythrocytes are presented in Table 1 . It is seen that not only the particle shape, but also the particle surface relief affected the power spectrum. This is an interesting result, as the spectrum of capacity does not contain information on surface microrelief of particles at small concentrations [5].

For another example of scattered particles exhibiting different geometry, we consider a potential of DWS for estimating the degree of aggregation of erythrocytes. Fig. 3 gives the normalized power $S(\omega)$ for the blood samples with both a low and a high degree of the erythrocyte aggregation. For the blood samples with relatively small aggregate sizes $(15-25 \mu \mathrm{m})$, we took normal blood from healthy donors, and used the blood of patients with cardiac ischemia for comparison. Blood of such patients is characterized by a higher degree of aggregation [16]. The average size of these larger aggregates is $70-150 \mu \mathrm{m}$.

The average size of the aggregates was monitored by microscope and by using an independent method, developed earlier [17]. This technique is based on measuring of the diffuse reflection and transmission coefficients at multiple scattering, and monitors the increase in the sizes of aggregates of whole blood at cardiac ischemia.

Fig. 4 presents the results to the halfwidth of spectrum changes with the increase in the concentration of oxygenated blood $(\mathrm{C} \%)$. It appears that by reduction of the blood oxygenation, it is possible to create a situation, when the halfwidth of spectrum for the disk-shaped particles will be even smaller than halfwidth of spectrum for the spherical particles (see Table 2). The oxygenation $\alpha$ was determined by the technique described in detail in Ref. [18].

It is necessary to note that the spectra, recovered in the experiments, change depending on sex, age, and disease. By averaging the results of the measurements into two groups, we obtained the average spectra for patients and healthy donors. The halfwidth changes of the spectrum for the samples with a strong degree of aggregation were in the range $1-4 \mathrm{kHz}$, and for the healthy donors, from 5 to $15 \mathrm{kHz}$. This is explained by reduction in speed of the aggregated erythrocytes at the increase of their sizes.

We suppose, the linear dependence of width of the spectrum of power $S(\omega)$ from the degree of oxygenation of erythrocytes of a blood uncloses possibility for the development of an express method of definition of density of oxygenation in erythrocytes of a blood.

In our experiments, we emphasized that the correlation coefficient between the halfwidth of spectrum depends on the lipoprotein concentration, whereas the relationship between the probability of falling sick and the other biochemical parameters is much weaker. Based on the relationship between the concentration of low-density lipoproteins and a possibility of cardiac ischemia disease [19], a new approach for quick diagnosis of cardiovascular diseases appears [20].

\section{Conclusion}

The experimental results clearly show that the PCS technique can be used to monitor the average characteristics of red blood cells under multiple scattering regime. This opens wide possibilities for the practical use of DWS in biomedical diagnosis by means of the monitoring of the shape of blood elements and their oxygenation. The experimental results presented above well illustrate the sensitivity of the method in its ability to monitor particle shape change and microcirculation.

\section{References}

[1] V.V. Tuchin (Ed.), Application in Medical Diagnostics and Therapy, SPIE Milestone Series, vol. MS 102, 1994.

[2] V.V. Tuchin, Light scattering study of tissues, Usp. Fiz. Nauk 167 (1997) 517-539.

[3] A.D. Lebedev, Yu.N. Levchyk, A.V. Lomakin, V.A. Noskin, Laser Correlation Spectroscopy in Biology, Navukova Dumka, Kiev, 1987.

[4] V.V. Tuchin, Laser and Fiber Optics in Biomedical Investigations, Saratov Univ. Press, Saratov, 1998.

[5] H.Z. Cummins, E.R. Pike, Photon Correlation and Light Beating Spectroscopy, Plenum, New York, 1973.

[6] R. Pecora, Dynamic Light Scattering: Applications of Photon Correlation Spectroscopy, Plenum, New York, 1985.

[7] W. Brown, Dynamic Light Scattering, The Method and Some Applications, Clarendon Press, Oxford, 1993.

[8] D.J. Pine, D.A. Weitz, G. Maret, P.E. Wolf, E. Herbolzheimer, P.M. Chaikin, Dynamical correlations of multiply scattered light, Scattering and Localization of Classical Waves in Random Media, Scattered Light, in: P. Sheng (Ed.), World Scientific Series on Direction in Condensed Matter Physics, vol. 8, World Scientific Publishing, 1990, pp. 312-372.

[9] A.G. Yodh, P.D. Kaplan, D.J. Pine, Pulse diffusing wave spectroscopy: high resolution through nonlinear optical gating, Phys. Rev. B 42 (1990) 4744-4752.

[10] I.V. Meglinsky, A.D. Boas, A.G. Yodh, B. Chance, In vivo measuring of blood flow changes using diffusing wave correlation techniques, in: R.R. Alfano, J.G. Fujimoto (Eds.), Advances in Optical Imaging and Photon Migration, vol. 2, 1996, pp. 195-197.

[11] I.V. Meglinsky, A.D. Boas, A.G. Yodh, B. Chance, V.V. Tuchin, Development of the correlation fluctuations techniques for the noninvasive monitoring and measurements parameters of blood flow, Izv. VUZ, Appl. Nonlinear Dyn. 4 (1996) 72-81. 
[12] V.V. Protopopov, N.D. Ustinov, Lasers Heterodyning, Nauka, Moscow, 1985.

[13] M. Born, E. Wolf, Principles of Optics: Electromagnetic Theory of Propagation, Interference and Diffraction of Light, Pergamon, New York, 1986.

[14] V.A. Levtov, S.A. Regirer, N.K. Shadrina, Rheology of Blood, Medicina, Moscow, 1982.

[15] V.L. Kuzmin, V.P. Romanov, Coherent phenomena in light scattering from disordered systems, Usp. Fiz. Nauk 166 (1996) 247-278.

[16] V.A. Losev, The mechanism of aggregation of erythrocytes at cardiac ischemia, Probl. Hematol. Blood Transfus. 24 (1979) 124129.
[17] G.S. Dubova, A.Ya. Khairullina, S.F. Shumilina, Reconstruction of the imaginary part of the refractive index of pigment of "soft" particles at their dense packing, J. Appl. Spectrosc. 34 (1984) $1058-1064$.

[18] A.Ya. Khairullina, Determination of oxygen percentage in whole blood, Vesti AN BSSR 6 (1984) 72-76.

[19] V.O. Shnikiter, T.N. Drozd, A.N. Klimov, Lipoproteins, BME 13 (1980) Minsk.

[20] A.N. Korolevich, N.P. Prigun, Assessment of the possibility of using dynamic spectroscopy for analyzing biological scattering media, J. Appl. Spectrosc. 64 (1997) 101-107. 